\title{
Inclusão de colesterol e seus efeitos na integridade das membranas plasmática e acrossomal de espermatozoides criopreservados de jumentos da raça Pêga
}

Flávia Vieira de Freitas ${ }^{[]^{*}}$, Maria Eduarda Borges Figueira, Cristian da Silva Teixeira ${ }^{[b]}$, Thiago Augusto Teles de Souza ${ }^{[b]}$, Carlos Mattos Teixeira Soares ${ }^{[b]}$, Maria Gazzinelli Neves ${ }^{[b]}$, Bruna Waddington de Freitas ${ }^{[b]}$, Larissa Marques de Oliveira ${ }^{[b]}$, Marina da Silva Passarelli[i], Mariana Andrade Torres ${ }^{[a]}$, Simone Maria Massami Kitamura Martins ${ }^{[a]}$, André Furugen Cesar de Andrade ${ }^{[a]}$, Giovanni Ribeiro de Carvalho[b]

\footnotetext{
[a] Universidade de São Paulo (USP), Pirassununga, SP, Brasil

[b] Universidade Federal de Viçosa (UFV), Viçosa, MG, Brasil
}

*Autor correspondente

e-mail: flavia.vet10@gmail.com

\section{Resumo}

A criopreservação do sêmen de diversas espécies, apesar de ser uma importante biotécnica reprodutiva, pode causar diversas lesões à célula espermática. A membrana plasmática do espermatozoide sofre a chamada fase de transição durante o abaixamento da temperatura, passando de um estado líquido-cristalino para o estado em gel. Durante a mudança de fase, é possível que ocorra perda de função da membrana devido a uma inadequada reorganização dos seus componentes, o que poderia comprometer diretamente a capacidade fertilizante do espermatozoide, através da lesão à própria membrana plasmática e à membrana acrossomal. Assim, a adição do colesterol à membrana plasmática de espermatozoides tem demonstrado ser uma técnica que, aliada a adequados protocolos de criopreservação, promove bons parâmetros celulares após o descongelamento. Isto é possível graças à capacidade do colesterol de abaixar a temperatura da fase de transição da membrana plasmática, mantendo esta estrutura fluída mesmo em baixas temperaturas e evitando a reorganização inapropriada de seus componentes. Desta forma, avaliou-se os efeitos da inclusão de colesterol na integridade das membranas plasmática e acrossomal de espermatozoide de jumentos. Foram utilizadas cinco colheitas de sêmen de cinco jumentos da raça Pêga, com idade entre 3 e 10 anos e peso médio de 300 Kg. Após as avaliações do sêmen in natura, este foi dividido em duas alíquotas, destinadas ao grupo controle (GC) e ao grupo tratado (GT). 0 sêmen do GC foi diluído em meio à base de leite em pó (Botu-Sêmen ${ }^{\circledR}$ - Botupharma Botucatu, SP, Brasil) em proporção 1:1, centrifugado a 600 g durante 15 minutos, ressuspendido com meio à base de gema de ovo (Botu-Crio ${ }^{\circledR}$ - Botupharma Botucatu, SP, Brasil) para obtenção de concentração de $200 \times 106$ espermatozoides/mL, envasado em palhetas francesas de 0,5 mL, refrigerado a 5을 durante 20 minutos, mantido a $4 \mathrm{~cm}$ do vapor de nitrogênio durante 15 minutos e mergulhado em nitrogênio líquido para congelamento do sêmen. 0 sêmen do GT foi previamente incubado com 1,5 mg de ciclodextrina (C4555 
- Sigma-Aldrich, St. Louis, Missouri, EUA) carregada com colesterol (C3045 - Sigma-Aldrich, St. Louis, Missouri, EUA) a cada 120 x 106 espermatozoides/mL durante 15 minutos à temperatura ambiente e protegido da luz. Após este tempo, o sêmen do GT foi diluído em meio à base de leite em pó (Botu-Sêmen ${ }^{\circledR}$ - Botupharma Botucatu, SP, Brasil) em proporção 1:1 e procedeu-se com as mesmas etapas de criopreservação do GC. Para avaliação da integridade das membranas plasmática e acrossomal, as palhetas foram descongeladas em banhomaria a $37^{\circ} \mathrm{C}$ durante 30 segundos, e seu conteúdo incubado com $1 \mu \mathrm{L}$ de cada uma das sondas fluorescentes, sendo elas Syto-59 (Molecular Probes Inc., Eugene, Oregon, EUA), Iodeto de Propídio (Sigma-Aldrich, St. Louis, Missouri, EUA) e FITC-PSA (Sigma-Aldrich, St. Louis, Missouri, EUA). As análises foram realizadas em aparelho de citometria de fluxo modelo BDAccuri C6 (Beckton-Dickeson, San Jose, USA). Os dados foram analisados pelo programa SAS (SAS Institute Inc., 2010). Houve efeito $(P<0,05)$ da inclusão de colesterol na manutenção da integridade das membranas plasmática e acrossomal, caracterizada pela maior população de células AIMI (acrossoma íntegro e membrana íntegra) para os espermatozoides do GT $(21,42 \pm 2,53)$ em relação ao GC $(12,73 \pm 1,96)$. É pertinente inferir que a adição de colesterol aos espermatozoides se mostrou eficaz em promover maior estabilização da membrana plasmática e acrossomal, possivelmente tendo sido capaz de preservar sua funcionalidade ao longo do processo de criopreservação. Conclui-se com o presente estudo que a adição de colesterol ao sêmen de jumentos da raça Pêga, na concentração utilizada, é recomendada para a preservação da integridade das membranas plasmática e acrossomal durante o processo de criopreservação.

Palavras-chave: Colesterol. Ciclodextrina. Membrana plasmática. 